\title{
Fruits and Vegetables Grading Using Digital Image Processing Techniques
}

\author{
Kavita Patil $^{1}$, Manisha Gawande ${ }^{2}$ \\ Student, Department of Electronics and Telecommunication Engineering, Sipna College of Engineering \& \\ Technology, Amravati. \\ Assistant Professor, Department of Electronics and Telecommunication Engineering, Sipna College of \\ Engineering \& Technology, Amravati.
}

\begin{abstract}
New safe and fast methods for grading of fruits have important place in agricultural economy. At the present time traditional grading methods have still been used broadly. But high costs and some inconsistencies guide post harvesting industry to automation applications in classification operations. Recently, enterprises incline towards to automation systems for increasing working capacity and decreasing working costs. Inconsistencies associated with manual grading decrease when automated grading systems are used. Thus, error rate and costs decrease while speed increases. As known; size, shape, color and tissue are base criteria in the classification process. In this study, automatic apple grading by size and color using digital cameras and computerized image processing techniques were studied. The assembled system has achieved basic tasks but it needs to be developed further.
\end{abstract}

Key words: Image process, Digital image process, Machine vision, Fruit classification

\section{INTRODUCTION}

\subsection{Summary of Literature}

In the studies of non destructive fruit classification apple, tomato, orange, pepper, potato was examined. To detect the fruit in front of camera, in some studies images taking from the camera are processing continuously on the other hand some studies use sensor Various studies have been done on the colors of fruit. Bern (2002), make color classification using RGB color components and CIE chromaticity with Matlab, make size classification with form factor and box structure methods. McClure (1988) works with white potatoes to detect size and shape information, Rehkugler and Throop (1986) works with "Red Delicious" apples to detect defects of apples. Monochrome camera was used in both studies. At the end of works greens and other scars of potatoes creases and blemishes (reddish brown) of apples couldn't detected with monochrome camera. Thomas and Connoly (1986) used pictures taken by RGB signals. If we look works in general Rehkugler (1986) able to detect 30 apples per minutes. While Bennedsen (2005) reach to $92 \%$ accuracy rate, $\mathrm{Li}$ (2002) reach $93 \%$ by artificial neural network approach.

\subsection{Fruit Classification}

Fruits classifies according to color, size and defect conditions after harvesting. So, quantity of produced fruits categorized and stocked can be known. Fruits can be find buyer in the market after categorization and price determination.

\subsection{Manual Classification}

In traditional classification, workers classifies fruits manually by fruits desired size. Basic disadvantages of this process are high costs, fruit damage may occur, workers not being objective while inspecting. High costs occur for grading workers. This cost is taken by farmer and returns to the consumer as price increase. Different workers can be classifying the same fruit to the different size class. This situation may decrease accuracy of grading process. On the other hand mixing good fruits among to bad and bad among to good 


\section{International Journal of Scientific Research in Engineering and Management (IJSREM)}

cause loss of money and product. Apple can easily vulnerable and damageable fruit as physically. Any wrong touch to the fruit may be cause crushes, rots and bruises. These conditions cause loss of money and product too.

\subsection{Automatic Classification}

Different methods can be use to classify size and color of fruit with machine vision. These methods based on detecting and processing color-shape feature of fruit. Processed images are obtained by digital image devices and computer. Obtained images become ready after some preprocessing. Images are processed at the decision phase by computer software that algorithm was previously prepared. Every inspected fruit is guides to the related area by controlled mechanism.

In experimental works certain amount of fruit was classified by workers and then same fruits classified by grading system. According to these work results, accuracy rate reach $69-75 \%$ depending on the kind of fruit. Accuracy rate can be increased to 94-98 \% by applying NNA (Neural network algorithm) approach (Nagata et al., 1997; Unay et al., 2002). In addition fruit prices in the markets may be reduced by using these systems. One of the indispensable element developing fruit classification systems is able to fast and accurate analysis of fruit. As mentioned above, process time varies depend on to the fruit type. Addition to this, according to some resources, a second three is enough for all processes (Nagata et al., 1997).

\section{MATERIAL AND METHOD}

System was developed as prototype in the experimental work. Created computer software shows apples size and color info taken images in the monitor according to system embedded sensors.

\section{Material}

Experimental mechanism consists of two parts; conveyor band and protective box. Box is located on the conveyor band and provides insulation against light conditions. Conveyor band carries fruits to the partially closed box. Sensor inside the box detects the fruit that come the relevant region. Camera takes image of fruit in front of the sensor and sends to the computer to process. System installation was started with mounting rubber band to the carrier frame. DC motor was used to ensure the movement of band.

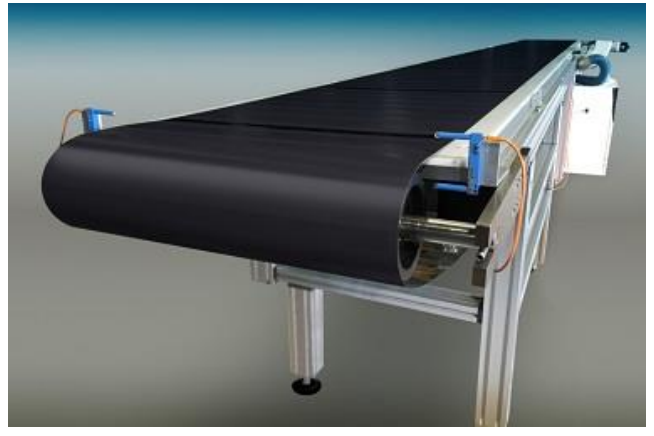

Figure 1. General view of conveyor band

\section{Method}

Software starts working with taking image from camera when take signal from sensor through parallel port. Processes were specified in the software algorithm. In the algorithm, firstly inspects apple's size and then color by using size information. After completion of transaction size and color information of apples shown in the monitor. This area did not cause insufficient light. Reason of this dark area is not to reflect coating material in the background. It's easy to gather only inspected object (apple) image with not to reflect light this material. In addition, camera can view moving band from sidelong easily with this approach.

In the studies, inspecting and evaluating of taken images from camera has been performed. Matlab program, Matlab Image Processing Toolbox, Image software and SigmaScan Software were used to makesense images for computer. Software algorithm was developed in the light of obtained data. Processes in the algorithm can be figures as follows.

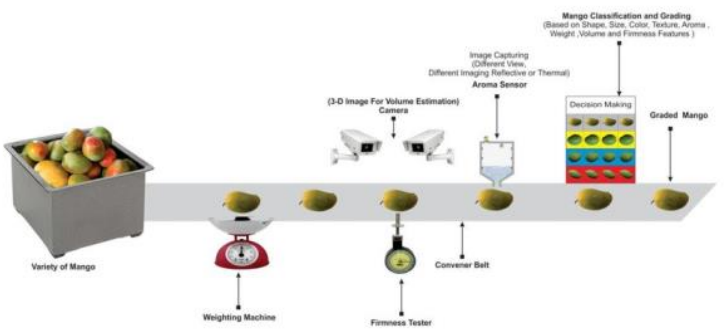

Figure 2. Overall functioning of the fruit grading system 


\section{International Journal of Scientific Research in Engineering and Management (IJSREM)}

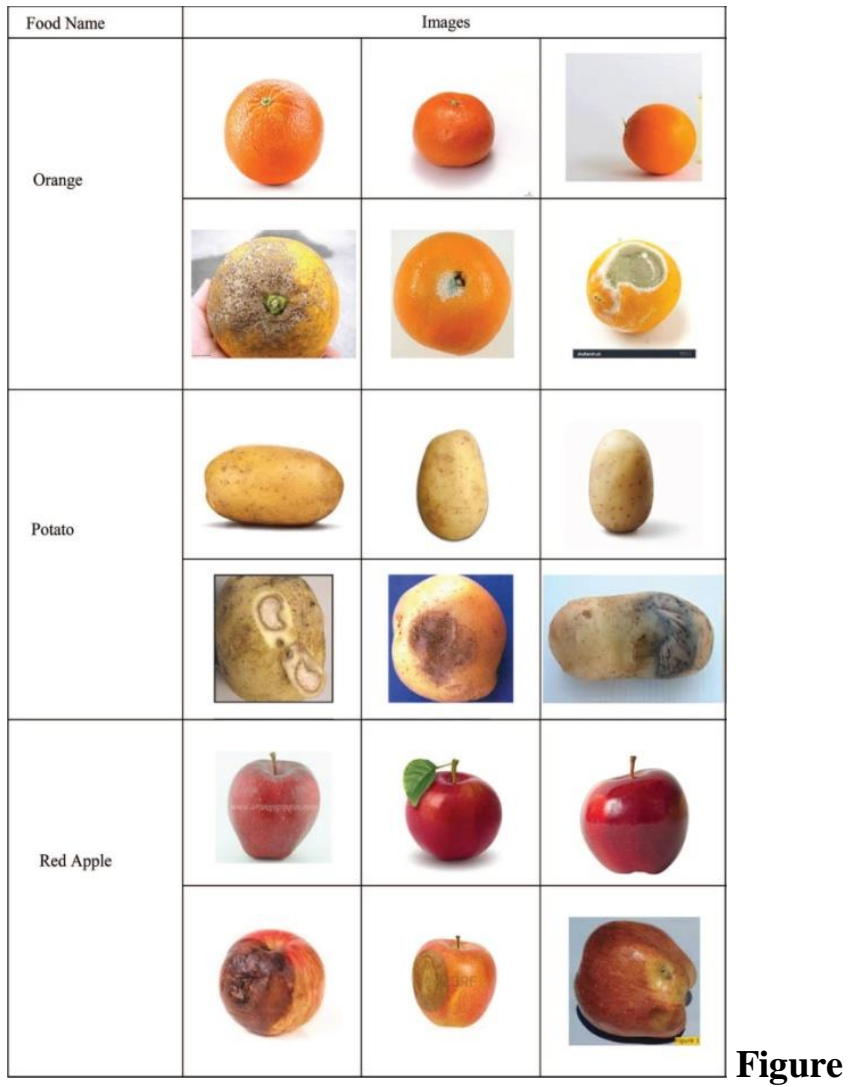

3. Inspecting different fruit with software

For shortening each images processing time process load tried to decreased by physically methods while experimental mechanism developing. In addition some arrangements made to increase software accuracy.

\section{Size Determination}

In size determination first, the necessary transformations made over RGB image of fruit. Edges of the fruit taken images are determined and marked as pixel over image. Area of the edge detected fruit provides information about the actual size is very close to real size.

Size of apple can be obtained by multiplying height and width values gathered from size inspection. Apples are divided into three classes and classified as small, medium and big after evaluation results.

\section{Color Determination}

One of the most important data in the process of color determination is edge values obtained from size determination process.
While determining color, size values calculated previous step are taken into account. Process is performed only on the rectangle area fruit cover on the image. This prevents the color determination algorithm to produce wrong result by taking action over unnecessary areas. Moreover, loss of time will also be reduced.

At the algorithm development stage histogram of pure apple images was extracted according to several color models. Detailed numerical analysis has been done on them. Green, yellow and red apple images inspected according to , RGB, HSB, Lab, XYZ, CMY color models. RBG color space is determined as more compatible method for color classification when processing time is taken into account. Software, classifies apples as yellow, red and green by doing necessary process according to data coming from camera on RGB color space. Figure 8 shows screenshot of software during running. This image shows that inspected apple is middle and yellow.

\section{CONCLUSION}

An important problem was tried to solve by making homogenous lighting inside the box and by preventing fruit illuminating directly (Yang, 1993; Penman, 2001). So, software prevented to wrong. First, the process determines the size. Color was determined with numerical data obtained here. Color of the image to determine the process by preventing unnecessary work in the area to increase the accuracy of the decisions and processes to reduce time is right. To evaluate the RGB, the color mode is based on computer media, in terms of working hours has been determined to be appropriate. Decision-making process similar to RGB color, other color modes are available. To use these color modes RGB values through the first to do some mathematical operations are needed. And this is a disadvantage to use this color mode. The system in the classification process has been addressed to reach the following objectives are required.

1. Systems analysis of the size and color,

2. The system is running in real time,

3. Just as the speed and accuracy rate of economic access. 


\section{REFERENCES}

1. Bennedsen, B.S., Peterson, D.L., Tabb, A., 2005, Identifying defects in images of rotating apples. Computers and Electronics in Agriculture, 48, 92-102.

2. Bern, A., Kuzivanov, I., 2002. Classification of Fruits. Machine Vision 2002, Course Report Project v0.1, 04.04.2002, Laboratory of Data Processing, Department of Information Technology, Lappeenranta University of Technology, 16s., Finland.

3. Delwiche, M. J., Tang, S., Thompson, J. F., 1993. A High Speed Sorting System for Dried Prunes. Transactions of the ASAE, 36(1), 195200. FAO, 2009. Food and Agriculture Organisation, FAOSTAT.

4. Haggar, R. J., Stent, C. J., Issac S., 1983. A Prototype Hand- Held Patch Sprayer for Killing Weeds, Activated by Spectral Differences in Crop/Weed Canopies. Journal of Agricultural Engineering Research, 28, 349-358. 